\title{
Atomic diffusion induced by stress relaxation in InGaAs/GaAs epitaxial layers
}

\author{
P. Roura ${ }^{\text {a) }}$ \\ GRM, Dept. Enginyeria Industrial, Escola Politècnica Superior, Universitat de Girona, \\ Av. Lluís Santaló s/n, E17071 Girona, Catalonia, Spain
}

\author{
A. Vilà, J. Bosch, M. López, A. Cornet, and J. R. Morante \\ E.M.E., Dept. Física Aplicada i Electrònica, Facultat de Física, Universitat de Barcelona, \\ Diagonal 645, E08028 Barcelona, Catalonia, Spain \\ D. I. Westwood \\ Department of Physics and Astronomy, University of Wales, College of Cardiff, P.O. Box 913, Cardiff, \\ CF2 3YB, Wales, United Kingdom
}

(Received 11 December 1996; accepted for publication 23 April 1997)

\begin{abstract}
The origin of the microscopic inhomogeneities in $\operatorname{In}_{x} \mathrm{Ga}_{1-x}$ As layers grown on GaAs by molecular beam epitaxy is analyzed through the optical absorption spectra near the band gap. It is seen that, for relaxed thick layers of about $2.8 \mu \mathrm{m}$, composition inhomogeneities are responsible for the band edge smoothing into the whole compositional range $(0.05<x<0.8)$. On the other hand, in thin enough layers strain inhomogeneities are dominant. This evolution in line with layer thickness is due to the atomic diffusion at the surface during growth, induced by the strain inhomogeneities that arise from stress relaxation. In consequence, the strain variations present in the layer are converted into composition variations during growth. This process is energetically favorable as it diminishes elastic energy. An additional support to this hypothesis is given by a clear proportionality between the magnitude of the composition variations and the mean strain. (C) 1997 American Institute of Physics. [S0021-8979(97)03715-8]
\end{abstract}

\section{INTRODUCTION}

The need of reliable high-quality devices for high frequency and optoelectronic applications requires the growth of III-V epitaxial layers, usually grown on InP and GaAs substrates. The layer composition was chosen so that its lattice parameter was the same as that of the substrate. The lattice-matched layers avoided the problems linked with the strain that develops when the lattice parameter is different. However, as strained layers of good quality are available through advanced epitaxial techniques, they are extensively studied because of their potential advantages. ${ }^{1}$

In any case (strained or matched), the layers are usually alloys of binary III-V semiconductors. This means that compositional homogeneity is not ensured. In fact, even with macroscopic homogeneous growing conditions over the whole wafer, microscopic compositional inhomogeneities are always present. In the literature, one can find two welldocumented origins of it. Apparently, even the best layers cannot avoid local statistical fluctuations of composition. ${ }^{2,3}$ This leads to local variations of the band gap reflected in the broadening of the free exciton peak observed by optical spectroscopies. Several authors have proposed that these fluctuations are the origin of a hyperfine contrast modulation (of the scale of a few $\mathrm{nm}$ ) in transmission electron microscopy (TEM). ${ }^{4}$ Yet TEM observations often reveal a contrast modulation with periodicity of hundreds of $\mathrm{nm}$ usually known as coarse modulation. ${ }^{5}$ The origin of these compositional inhomogeneities is usually related to thermodynamical

${ }^{a)}$ Electronic mail: pereng@EI.udg.es instabilities of the alloy that tends to break it down into its components (spinodal decomposition). ${ }^{6}$

Of course, these two cases do not cover all possible situations. For instance, linked with structural defects such as dislocations or stacking faults the exciton peak broadens. This broadening is usually taken as a quick evaluation of the layer quality and is a routine test. However, the lack of a technique which is sensitive to the microscopic origin of the broadening prevents a detailed analysis.

Recently, we have developed a technique that enables the question of whether the broadening is related to composition or strain inhomogeneities to be solved. ${ }^{7}$ It has been successfully applied to the case of partially relaxed InGaAs layers grown on $\operatorname{InP}^{8}$ We have been able to quantify the strain field due to local deviations from the mean density of dislocations. Now we propose to analyze the case of highly relaxed layers, namely, InGaAs/GaAs layers with $0.1<x$ $<0.9$. Contrary to what we expected a priori, the great density of dislocations does not result in a layer with great strain inhomogeneities; rather, the dominant inhomogeneities are compositional. The analysis shows that, during growth, the atoms arriving at the layer surface diffuse in order to minimize the elastic energy. The result is the diminution of the strain inhomogeneities and the appearance of composition variations.

\section{EXPERIMENT}

We have analyzed two sets of InGaAs epitaxial layers grown by molecular beam epitaxy on GaAs at $350{ }^{\circ} \mathrm{C}$. The growth conditions are detailed in Ref. 9. The first set is a series of thick layers with thickness of about $2.8 \mu \mathrm{m}$ and 




FIG. 1. OA spectra of thick layers $(2.8 \mu \mathrm{m}$ width $)$ measured at low temperature. The horizontal bar indicates the energy scale.

indium molar compositions varying from about $x=0.05$ up to $x=0.8$. In the second set, the composition is constant ( $x$ $\simeq 0.18$ ) but the thickness varies from 0.06 to $2.8 \mu \mathrm{m}$.

The optical absorption (OA) spectra were measured at low temperature $(\approx 10 \mathrm{~K})$ with the samples inside a continuous-flow He cryostat. The transmitted light was detected with an InAs or an InSb photodiode depending on the spectral region.

\section{RESULTS}

\section{A. Optical absorption measurements}

For every layer, we have measured its optical absorption near the band gap. The resulting spectra are shown in Fig. 1 for the thick layers and in Fig. 2 for the layers of variable thickness. In Fig. 1 the great energetic shift of the absorption edge is mainly due to the dependence of the band gap energy on the indium molar composition $x$, (Ref. 10)

$$
E_{g 0}=1.519-1.608 x+0.5 x^{2}(\mathrm{eV})(4 \mathrm{~K}),
$$

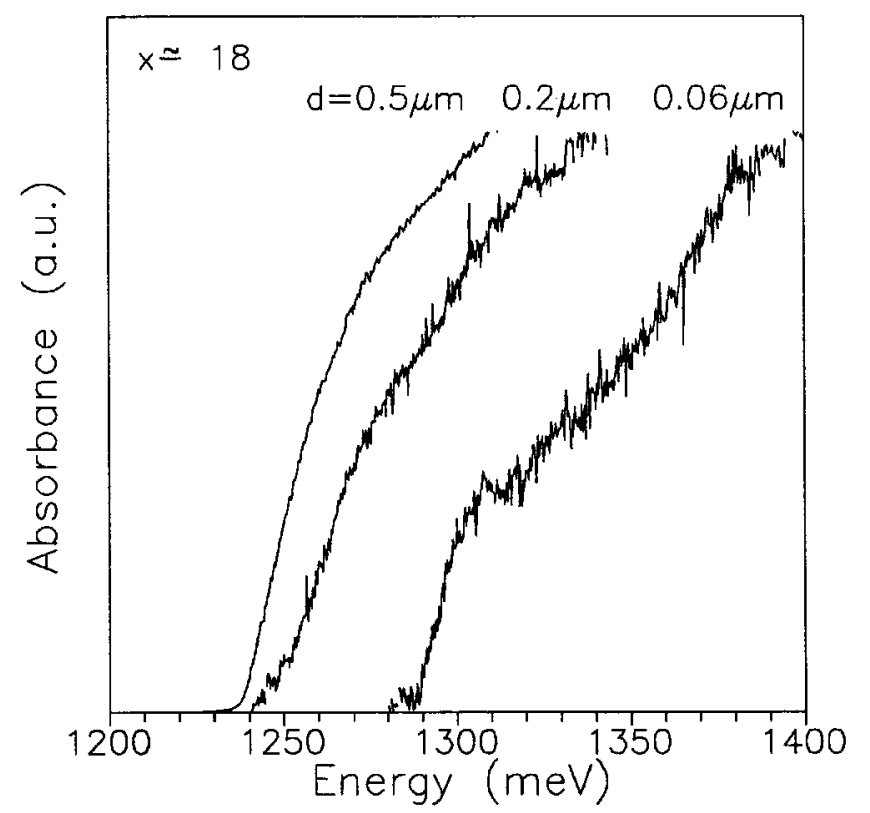

FIG. 2. OA spectra of layers with different thicknesses and similar composition.

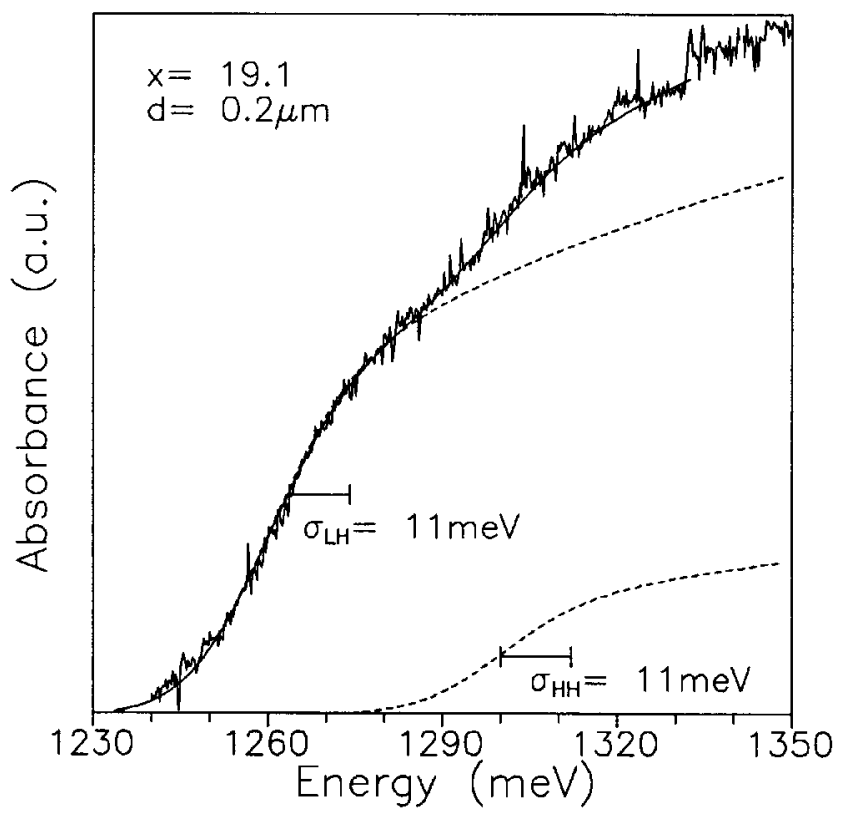

FIG. 3. Experimental (solid line) and calculated (broken line) OA spectra for a sample with dominant compositional inhomogeneities. The contributions of the $\mathrm{HH}$ and $\mathrm{LH}$ subbands are indicated to show that their energetic dispersions are equal.

where $E_{g 0}$ is the value of the unstrained band gap. However, as the layers are strained, there is an additional shift due to the biaxial strain $\epsilon$ that can be quantified according to ${ }^{11}$

$$
\Delta E_{\mathrm{LH}}=\Delta_{\mathrm{LH}} \epsilon,
$$

where

$$
\Delta_{\mathrm{LH}}(x)=1.53 x+6.26(1-x) .
$$

The subindex LH stands for "light hole" because, as the layers are compressed, the band gap is determined by this subband whereas the subband of the heavy holes $(\mathrm{HH})$ lies at higher energy. The existence of a strain is clearly revealed by the splitting of the band edge absorption that can be observed in every spectrum. In Fig. 2, as the composition of the samples is held constant, the built-up strain is the only parameter that induces the shift and splitting of the band edge. We can observe in Fig. 2 a progressive diminution of both the band gap and the splitting. This indicates that, as thickness increases, the strain relaxes. In every sample the strain can be easily quantified from the splitting, $\Delta E$, because ${ }^{11}$

$$
\Delta E=\left(\Delta_{\mathrm{LH}}-\Delta_{\mathrm{HH}}\right) \epsilon,
$$

where

$$
\Delta_{\mathrm{HH}}=9.04 x+12.74(1-x) .
$$

So, the molar composition $x$ and the mean strain $\epsilon$ can be obtained from the band gap energy $E_{g}$ and the splitting $\Delta E$, which, in turn, are directly extracted from the OA spectra by fitting. The procedure is detailed in Ref. 11. Here we just want to show the good agreement between the experimental and the calculated spectra (Figs. 3 and 4). In both figures we have detailed the structure of the spectrum by plotting the $\mathrm{HH}$ and $\mathrm{LH}$ subbands separately. The results of 




FIG. 4. Experimental (solid line) and calculated (broken line) OA spectra for the thinnest sample of this study. Its dominant microscopic inhomogeneities are of strain. It is clearly seen that the energetic dispersion of the $\mathrm{HH}$ subband is much greater than that of the LH subband.

the fitting are summarized in Tables I and II for the two sets of samples.

\section{B. Quantification of composition inhomogeneities}

Local variations of strain or composition involve local variations of band gap energy. This is reflected as a smoothing of the optical absorption edge. To take this effect into account, the $\mathrm{HH}$ and $\mathrm{LH}$ subbands of the calculated spectrum are convoluted with Gaussian distributions characterized by its standard deviations, $\sigma_{\mathrm{HH}}$ and $\sigma_{\mathrm{LH}}$. The values needed to obtain good fitting are detailed in Tables I and II.

We have demonstrated in a previous work $^{7}$ that the nature of microscopic inhomogeneities can be easily found through the value of $\sigma_{\mathrm{HH}} / \sigma_{\mathrm{LH}}$. If $\sigma_{\mathrm{HH}} / \sigma_{\mathrm{LH}} \leqslant 1$, they correspond to compositional variations; whereas if $\sigma_{\mathrm{HH}} / \sigma_{\mathrm{LH}}>1$ (in fact $\sigma_{\mathrm{HH}} / \sigma_{\mathrm{LH}}=\Delta_{\mathrm{HH}} / \Delta_{\mathrm{LH}}$, e.g., 2.0 for $x \approx 0$ and 5.9 for $x \approx 1$ ), they are due to local variations of strain. To be more exact, this criterium was established by considering that the local variations of strain did not affect its biaxial character. However, a detailed work that analyzed by cathodoluminescence the local stress relaxation in $\mathrm{InGaAs} / \mathrm{GaAs}$ epitaxial layers $^{12}$ clearly showed the existence of regions with a great uniaxial character. This somewhat more complicated situation can be addressed both theoretically and experimentally. It can be simply demonstrated that the tetragonal distortion is more important with a uniaxial strain. As this distortion has a different effect on the energy of the HH and LH subbands, the resulting $\sigma_{\mathrm{HH}} / \sigma_{\mathrm{LH}}$ ratio would be even greater than with a pure biaxial strain. From the experimental point of view, our results on partially relaxed $\mathrm{InGaAs}$ layers ${ }^{8}$ confirm this theoretical analysis. On samples $\mathrm{C}$ and $\mathrm{D}$ the dislocation densities along the [110] directions were twice the density along the perpendicular direction. This anisotropic relaxation would imply, as shown in Ref. 12, a local uniaxial character of the strain. Even in these samples, the value of $\sigma_{\mathrm{HH}} / \sigma_{\mathrm{LH}}$ remained clearly greater than 1 .

Now, from Table I we conclude that the dominant inhomogeneities in the thick layers are compositional $\left(\sigma_{\mathrm{HH}} / \sigma_{\mathrm{LH}}\right.$ $\approx 1)$. Furthermore, from $\sigma_{\mathrm{LH}}$ we can obtain the standard deviation of the composition distribution $\sigma_{x}$. From the dependence of $E_{g 0}$ with $x$ [Eq. (1)], it follows

$$
\sigma_{E}=(-1.608+x) \sigma_{x} \text {. }
$$

This equation has been applied in those samples where $\sigma_{\mathrm{HH}} / \sigma_{\mathrm{LH}} \leqslant 1$. In the remaining samples $\left(\sigma_{\mathrm{HH}} / \sigma_{\mathrm{LH}}\right.$ slightly greater than 1) a correction has been introduced to account for the small contribution of the strain inhomogeneities. The value of $\sigma_{x}$ is quoted in the last column of Table I.

In the case of the set of variable thickness (Table II) we see that composition inhomogeneities dominate in the thicker samples $\left(\sigma_{\mathrm{HH}} / \sigma_{\mathrm{LH}}=1\right)$, whereas in the thinner one the spectral shape (Fig. 4) is in line with the existence of strain variations $\left(\sigma_{\mathrm{HH}} / \sigma_{\mathrm{LH}}=2.4\right)$. This result agrees with the measurements done on InGaAs/GaAs thin layers of different origin where dominant inhomogeneous strain variations were also found.

\section{ANALYSIS: THE ORIGIN OF THE COMPOSITION INHOMOGENEITIES}

\section{A. Problem statement}

Here the origin of the compositional inhomogeneities observed in thick layers will be analyzed (Tables I and II).

TABLE I. Layer parameters of thick samples: In molar composition $(x)$, band gap energy $\left(E_{g}\right)$, valence band splitting $(\Delta E)$, standard deviation of the heavy-hole and light-hole subbands $\left(\sigma_{\mathrm{HH}}, \sigma_{\mathrm{LH}}\right)$, mean strain $(\epsilon)$, and standard deviation of the molar composition $\left(\sigma_{x}\right)$. The energies are given in $\mathrm{meV}$.

\begin{tabular}{|c|c|c|c|c|c|c|}
\hline$x(\%)$ & $E_{g}$ & $\Delta E$ & $\sigma_{\mathrm{LH}}$ & $\sigma_{\mathrm{HH}} / \sigma_{\mathrm{LH}}$ & $\epsilon\left(10^{-3}\right)$ & $\sigma_{x}(\%)$ \\
\hline 4.0 & 1455.7 & 12.0 & 3.5 & 1.1 & 1.85 & 0.22 \\
\hline 11.2 & 1345.5 & 10.0 & 3.5 & 1.0 & 1.57 & 0.23 \\
\hline 16.9 & 1261.2 & 11.8 & 3.2 & 1.0 & 1.88 & 0.22 \\
\hline 23.9 & 1164.0 & 16.0 & 4.0 & 1.0 & 2.57 & 0.29 \\
\hline 34.1 & 1029.5 & 20.0 & 6.0 & 1.0 & 3.26 & 0.47 \\
\hline 43.7 & 911.5 & 21.5 & 6.2 & 1.0 & 3.57 & 0.54 \\
\hline 53.2 & 805.0 & 27.0 & 8.0 & 1.0 & 4.56 & 0.75 \\
\hline 63.2 & 702.0 & 19.0 & 5.8 & 0.9 & 3.25 & 0.61 \\
\hline 71.6 & 623.8 & 15.0 & 4.5 & 1.4 & 2.61 & 0.42 \\
\hline 81.1 & 543.7 & 11.7 & 3.9 & 1.2 & 2.07 & 0.46 \\
\hline
\end{tabular}


TABLE II. Layer parameters of samples with various thicknesses: In molar composition $(x)$, layer thickness $(d)$, band gap energy $\left(E_{g}\right)$, valence band splitting $(\Delta E)$, standard deviation of the heavy-hole and light-hole subbands $\left(\sigma_{\mathrm{HH}}, \sigma_{\mathrm{LH}}\right)$, mean biaxial strain $(\epsilon)$, standard deviation of the molar composition, and strain $\left(\sigma_{x}\right.$ and $\left.\sigma_{\epsilon}\right)$. The energies are given in meV.

\begin{tabular}{ccccccccc}
\hline \hline$x(\%)$ & $d(\mu \mathrm{m})$ & $E_{g}$ & $\Delta E$ & $\sigma_{\mathrm{LH}}$ & $\sigma_{\mathrm{HH}} / \sigma_{\mathrm{LH}}$ & $\epsilon\left(10^{-3}\right)$ & $\sigma_{x}(\%)$ & $\sigma_{\epsilon}\left(10^{-3}\right)$ \\
\hline 18.4 & 0.06 & 1293.5 & 68 & 5.0 & 2.4 & 10.8 & - & 0.76 \\
19.1 & 0.2 & 1262.5 & 40 & 11.0 & 1.0 & 6.4 & 0.78 & - \\
17.9 & 0.5 & 1250.0 & 20 & 8.0 & 1.0 & 3.2 & 0.56 & - \\
16.9 & 2.8 & 1261.2 & 11.8 & 3.2 & 1.0 & 1.9 & 0.23 & - \\
\hline \hline
\end{tabular}

The high values of $\sigma_{\mathrm{LH}}$ mean that it cannot be due to statistical fluctuations. Maximum fluctuations would be at $x$ $=0.5$ and for InGaAs would give $\sigma_{\mathrm{LH}} \approx 0.69 \mathrm{meV},{ }^{13}$ which is much less than the values of Tables I and II. On the other hand TEM observations have not revealed any contrast modulation that would suggest the existence of spinodal decomposition. So, we have to find an alternative mechanism to explain the composition inhomogeneities.

In fact, there is a more striking question still unresolved: why are the dominant inhomogeneities not due to strain? Samples are relaxed and the remaining strain could change locally due to local deviations from the mean density of dislocations. This effect has been identified in partially relaxed InGaAs layers ${ }^{8}$ and, presumably, is the origin of the strain inhomogeneities observed in the thinnest sample of this study (Table II). So, why does it not appear in highly relaxed layers?

\section{B. Atomic diffusion induced by strain}

The key to identifying a mechanism that gives rise to the dominant composition variations is probably the evolution

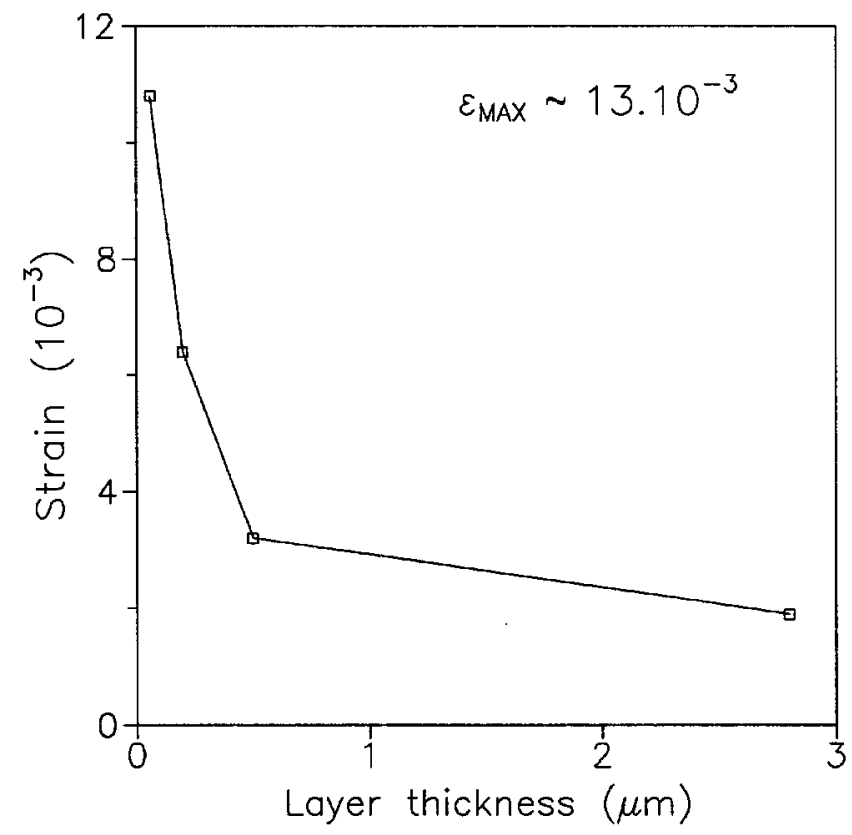

FIG. 5. Evolution of strain with layer thickness. For the thickest layer (2.8 $\mu \mathrm{m})$ most of the relaxation has occurred at a sublayer of about $0.5 \mu \mathrm{m}$. The maximum strain quoted corresponds to $x=0.18$. It has to be taken as only approximate because the composition changes slightly from sample to sample. observed as the layer thickness increases. We think that the strain inhomogeneities detected in the thinnest sample are responsible for the composition inhomogeneities that develop as the growth proceeds. The mechanism would be the atomic diffusion induced by strain gradients at the layer surface. The driving force of this process, as will be shown, is the reduction of the elastic energy of the layer. To understand this, let us describe the ideal structure of highly relaxed layers. From the molar composition, we can easily calculate the maximum strain of an $\operatorname{In}_{x} \mathrm{Ga}_{1-x}$ As layer grown on GaAs

$$
\epsilon_{\mathrm{MAX}}=\frac{a_{\mathrm{GaAs}}-a_{\mathrm{InGaAs}}}{a_{\mathrm{InGaAs}}} \approx 0.072 x(4 \mathrm{~K}),
$$

where $a_{\mathrm{GaAs}}$ and $a_{\mathrm{InGaAs}}$ are the lattice parameters. ${ }^{11}$ This would be the strain of layers thin enough to prevent relaxation. In the case of the set of thick layers, the remaining strain, $\epsilon$ (Table I), is less than $20 \%$ of $\epsilon_{\mathrm{MAX}}$. TEM observations ${ }^{9}$ show a great density of dislocations in a sublayer near the interface, whereas it diminishes in the remaining upper layer. This means that most of the strain relaxes before growth has finished. This is supported by the evolution of strain in the layers of increasing thickness (Fig. 5) ${ }^{14}$ and by the theories on the relaxation mechanisms. ${ }^{15}$

The relaxed sublayer will have a strain distribution $\sigma_{\epsilon}$ due to local variations in the dislocations density. Now, if the upper layer were homogeneous in composition, it would reproduce the strain variations of the sublayer. This would result in an excess of elastic energy $\Delta U_{\text {elastic }}$ compared to a layer with homogeneous strain

$$
\Delta U_{\text {elastic }} \propto \sigma_{\epsilon}^{2} .
$$

This excess of energy can be avoided if the atoms arriving onto the surface migrate in order to compensate for the strain variations. So, the initially more compressed regions will become enriched in GaAs and the strain distribution of the sublayer will become converted into composition variations in the upper layer (most of the layer volume).

In view of the real evolution observed in the layers of increasing thickness, some refinements can be introduced into the above description. The relaxed sublayer does not have a homogeneous composition through its entire thickness. We see in Fig. 5 that the thinnest layer $(0.06 \mu \mathrm{m})$ has relaxed only around $20 \%$ of the maximum strain and that the following layer in the series $(0.2 \mu \mathrm{m})$ already has dominant composition inhomogeneities, although it is far from being completely relaxed. So, below a certain critical thickness, the atomic diffusion is not activated because the density of dis- 


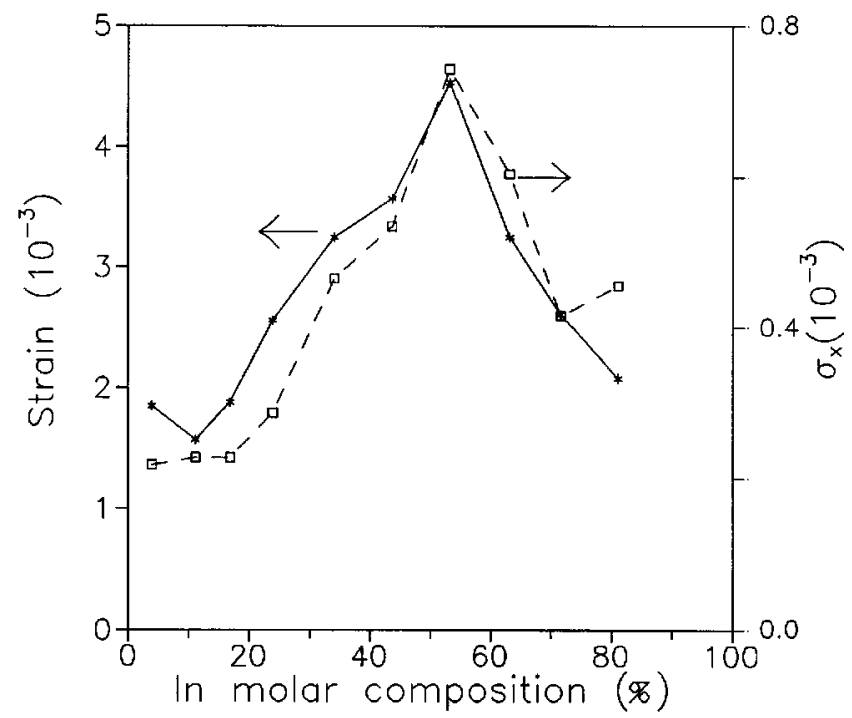

FIG. 6. Evolution of the mean strain $\epsilon$ and the composition standard deviation $\sigma_{x}$ with the molar composition.

locations has not yet developed. Up to this thickness the layer is homogeneous in composition. Above this thickness, atomic diffusion ("erases") both the strain inhomogeneities related to the initial relaxation and those related with the continuous increase in relaxation that develops as the layer grows.

A simple test can be proposed into this hypothesis. As a compressed layer cannot become tensed due to relaxation, we can establish an upper limit for the standard deviation of the strain $\sigma_{\epsilon}$

$$
\sigma_{\epsilon}<\epsilon_{\text {sublayer }}
$$

where $\epsilon_{\text {sublayer }}$ is the mean strain in the sublayer. It will be similar to the value of the whole layer, $\epsilon$. As the strain distribution is converted into a composition distribution, the resulting value of $\sigma_{x}$ will be related to $\sigma_{\epsilon}$. From the variation of the lattice parameter with $x$ and Eq. (7) we can establish an upper limit for $\sigma_{x}$

$$
\sigma_{x}<\sigma_{x}(\epsilon) \equiv-\frac{\epsilon}{0.072} .
$$

In all layers this condition is largely fulfilled. The value of $\sigma_{x}$ in the thick layers (Table I) is about ten times smaller than the upper value, $\sigma_{x}(\epsilon)$. For the other layers (Table II) the ratio is even greater.

Up to this point, we can simply state that the experimental evolution of microscopic inhomogeneities with layer thickness is as expected with the mechanism proposed and that the value of the composition variations is not in contradiction with it. However, proof that the composition inhomogeneities are really linked with the relaxation (or the strain) in the layers is required. This will be given in the next section.

\section{Correlation between $\sigma_{x}$ and strain}

In Fig. 6 we have plotted $\sigma_{x}$ and the mean strain, $\epsilon$, versus molar composition. They are roughly proportional throughout the whole composition range (this relationship is remarkably well followed below $x=0.6$ ). At this point it should be noted that, although both parameters are obtained through the fitting of the OA spectra, their effects on the spectral shape are quite independent. Consequently, their relationship is not an artifact due to the fitting procedure.

Additionally, we have tried to find a correlation with the density of dislocations or the broadening of the x-ray diffraction peak. ${ }^{9}$ Although those parameters also have a maximum around $x=0.5$, no quantitative relationship can be established with $\sigma_{x}$. They are simply complementary evaluations of the layer quality.

The correlation between $\sigma_{x}$ and strain was not expected. In fact, from the hypothesis of atomic diffusion we can only establish an upper limit for $\sigma_{\epsilon}$ [Eq. (10)]. The proportionality found with $\epsilon$ is similar to that found in partially relaxed samples ${ }^{8}$ where $\sigma_{\epsilon}$ was proportional and nearly equal to the relaxed strain. Apparently, when the layers are slightly or highly relaxed, there is a relationship between the mean strain and $\sigma_{\epsilon}$ that would convert equation (10) into

$$
\sigma_{\epsilon} \approx a \epsilon_{\text {sublayer }} \text { where } a<1 \text {. }
$$

The value of $a$ depends on the smoothing effect of the exciton. Short range variations of the strain are smoothed by the finite volume of the exciton as it occurs in quantum wells. ${ }^{16}$ This is the case of dislocations in the sublayer (in our case $a \approx 1 / 7)$. On the other hand, this smoothing effect is absent with a low density of dislocations. This is the case of the partially relaxed layers of Ref. 8 where $a \approx 1$.

Independently of why these two parameters are proportional, the good correlation between them indicates that $\sigma_{x}$ is related to the remaining strain. This relationship is not surprising if $\sigma_{x}$ arises from the strain inhomogeneities in the relaxed sublayer. So, this relationship reinforces the hypothesis of atomic diffusion induced by strain.

\section{CONCLUSIONS}

We have reported optical absorption measurements on highly relaxed $\mathrm{In}_{x} \mathrm{Ga}_{1-x}$ As $(0.05<x<0.8)$ layers grown on GaAs and layers of $x \approx 0.18$ with various thicknesses ranging from 0.06 to $2.8 \mu \mathrm{m}$.

The relative energy dispersion of the $\mathrm{HH}$ and $\mathrm{LH}$ subbands indicates that the dominant inhomogeneities in highly relaxed layers are due to composition variations. To explain this, it is proposed that during growth the atoms diffuse over the free surface in order to minimize the elastic energy. This mechanism would convert the initial strain inhomogeneities into composition variations. This hypothesis is supported by: (a) the absence of strain inhomogeneities in samples with a great density of dislocations; (b) the change from strain into composition inhomogeneities as the layers get thicker, and (c) the correlation between the composition variations and the mean strain.

Diffusion induced by strain is a well-known mechanism in Materials Science that accounts for deformation at high temperatures (the phenomenon known as creep). ${ }^{17}$ Like any diffusion mechanism, its dynamics is governed by a gradient and is thermally activated. This means that in the case of epitaxial layers, as described in this paper, the conversion of 
the strain into composition inhomogeneities will depend not only on the density of dislocations but also on a compromise between temperature and growth rate.

\section{ACKNOWLEDGMENTS}

This work has been supported by the Spanish Programa Nacional de Materiales under Contract No. MAT93-0564 and the HCM Strained Semiconductor Structures and Devices Program CHRX-CT94-0428.

${ }^{1}$ G. C. Osbourn, J. Vac. Sci. Technol. B 4, 1423 (1986).

${ }^{2}$ E. F. Schubert, E. O. Gobel, Y. Horikoshi, K. Ploog, and H. J. Queisser, Phys. Rev. B 30, 813 (1984).

${ }^{3}$ J. M. Langer, R. Buczko, and A. M. Stoneham, Semicond. Sci. Technol. 7, 547 (1992).

${ }^{4}$ T. Y. Seong, G. R. Booker, and A. G. Norman, AIP Conf. Ser. 134, 301 (1990); F. Glas, AIP Conf. Ser. 134, 269 (1990); F. Peiro, A. Cornet, and J. R. Morante, AIP Conf. Ser. 134, 291 (1990).

${ }^{5}$ P. Henoc, A. Izrael, M. Quillec, and H. Launois, Appl. Phys. Lett. 40, 963 (1982).

${ }^{6}$ B. de Cremoux, J. Phys. C 43, 5 (1982).
${ }^{7}$ P. Roura, J. Bosch, and J. R. Morante, Phys. Rev. B 46, 10453 (1992).

${ }^{8}$ P. Roura, S. A. Clark, J. Bosch, F. Peiro, A. Cornet, and J. R. Morante, J. Appl. Phys. 77, 4018 (1995).

${ }^{9}$ D. I. Westwood, D. A. Woolf, A. Vila, A. Cornet, and J. R. Morante, J. Appl. Phys. 74, 1731 (1993).

${ }^{10}$ D. K. Gaskill, N. Bottka, L. Aina, and M. Mattingly, Appl. Phys. Lett. 56, 1269 (1990).

${ }^{11}$ S. A. Clark, P. Roura, J. Bosch, A. Perez-Rodriguez, J. R. Morante, D. I. Westwood, and R. H. Williams, J. Appl. Phys. 77, 3393 (1995).

${ }^{12}$ K. Rammohan, D. H. Rich, R. S. Goldman, J. Chen, H. H. Wieder, and K. L. Kavanagh, Appl. Phys. Lett. 66, 869 (1995); K. Rammohan, Y. Tang, D. H. Rich, R. S. Goldman, H. H. Wieder, and K. L. Kavanagh, Phys. Rev. B 51, 5033 (1995).

${ }^{13}$ C. Charreaux, G. Guillot, and A. Nouailhat, J. Appl. Phys. 60, 768 (1986).

${ }^{14}$ This kind of evolution is similar to that found in other materials. See, for instance, D. J. Olego, Y. Okuno, T. Tawano, and M. Tamura, J. Appl. Phys. 71, 4502 (1992).

${ }^{15}$ J. W. Matthews and A. E. Blakeslee, J. Cryst. Growth 27, 118 (1974); J. H. van der Merwe, Surf. Sci. 31, 198 (1988).

${ }^{16}$ C. A. Warwick, W. Y. Jan, and A. Ouzmard, Appl. Phys. Lett. 56, 2666 (1990); M. A. Herman, D. Bimberg, J. Christen, J. Appl. Phys. 70, R1 (1991).

${ }^{17}$ G. E. Dieter, Mechanical Metallurgy, 3rd ed. (McGraw-Hill, New York, 1986), Chap. xxv. 